\title{
Oscillation of Cytoplasmic pH of Physarum Plasmodium in Relation to Motility
}

\author{
Soichi Nakamura and Noburô Kamiya \\ Department of Cell Biology, National Institute for Basic Biology, Okazaki 444, \\ Japan
}

\begin{abstract}
Short-term changes in the cytoplasmic pH in the plasmodium of Physarum polycephalum were measured with special reference to its contraction-relaxation cycle. The measurement was done photometrically in a plasmodial strand segment loaded with fluorescein diacetate. To eliminate possible errors caused by volume changes of the plasmodial strand in the course of its contraction-relaxation cycle, fluorescence intensities at two excitation wavelengths, $493 \mathrm{~nm}$ ( $\mathrm{pH}$ sensitive) and $448 \mathrm{~nm}$ ( $\mathrm{pH}$ insensitive), were recorded alternately. Simultaneously, tension production of the same strand segment was recorded under isometric conditions. The results were: 1) The cytoplasmic $\mathrm{pH}$ averaged $6.6 \pm 0.5$, for 34 specimens. 2) The cytoplasmic $\mathrm{pH}$ changed cyclically with the same period as the tensile force. 3) The average peak-to-peak amplitude of $\mathrm{pH}$ change was $0.13 \pm 0.06 \mathrm{pH}$ unit. 4) The phase of the $\mathrm{pH}$ oscillation was just opposite to that of the force production cycle; i.e., the cytoplasmic $\mathrm{pH}$ decreased during the contraction phase and increased during the relaxation phase.
\end{abstract}

The plasmodia of myxomycetes, especially of Physarum polycephalum, are among the most extensively investigated materials in cell motility research. However, only a few reports have appeared on the $\mathrm{pH}$ of Physarum plasmodia. Seifriz and Zetzmann (12) first estimated the cytoplasmic $\mathrm{pH}$ in Physarum by using the color changes of its intrinsic pigments as an indicator, with particular reference to its fruiting and sclerotium formation. The change in the cytoplasmic $\mathrm{pH}$ of Physarum in relation to its mitotic cycle was studied by Gerson and Burton (3) and Morisawa and Steinhardt (9). According to these authors, the $\mathrm{pH}$ rises at or near the stage of mitosis and reaches a low point at the mid-interphase of the mitotic cycle. Hirose et al. (5) used fluorometry of the intrinsic fluorescent pigments of Physarum to study the role of intracellular $\mathrm{pH}$ in chemotactic transduction and suggested that the degree of cytoplasmic acidification paralleled the relaxing phase of the tension cycle. However, there has been no report on the short-term $\mathrm{pH}$ changes in relation to the contractionrelaxation cycle.

In the present work, we simultaneously measured cytoplasmic $\mathrm{pH}$ and isometric tensile force production in a plasmodial strand segment of Physarum. The cytoplasmic $\mathrm{pH}$ was measured using a fluorescent $\mathrm{pH}$-indicator dye, fluorescein diacetate, which shows $\mathrm{pH}$-sensitive or -insensitive fluorescence excitation depending on the wavelength of the excitation light. 


\section{MATERIALS AND METHODS}

Plasmodia. Plasmodia of Physarum polycephalum (strain Ng-1), which had been cloned at the National Institute for Basic Biology from a sample supplied by Dr. S. Hatano of Nagoya University, was cultured by feeding with rolled oats in a large bucket in a modification of Camp's method (2).

Loading plasmodia with fluorescein diacetate. The fluorescent pH-indicator dye, fluorescein diacetate, diffuses into the cell, where it reacts with intracellular esterase, hydrolytically removing the acetate groups and thus releasing the charged chromophores into the cytoplasm. The strong negative charge of the chromophores inhibits their release from the cell. One of the important merits of this method is that the emission spectrum of the chromophore is highly dependent on $\mathrm{pH}(13,14)$. This high $\mathrm{pH}$ sensitivity allowed us to follow changes in $\mathrm{pH}$.

To load the plasmodial segment with fluorescein, a piece of dried sclerotium (ca. $1 \mathrm{~cm}^{2}$ ) on a filter paper was placed on a $1.5 \%$ agar plate containing $17 \mu \mathrm{M}$ fluorescein diacetate and left for $48 \mathrm{~h}$ in the dark without being fed. Under these conditions the reactivated plasmodium formed a network of ramifying strands, and its color changed slightly from yellow to green because of the incorporated fluorescein.

For tensiometry and fluorometry, a segment, $10-15 \mathrm{~mm}$ long and $80-200 \mu \mathrm{m}$ in diameter, of the smooth strand loaded with fluorescein was excised by cutting off the smaller branches from the network region on the agar surface.

Simultaneous measurement of tensile force and fluorescence. To record the cyclic tension generation of an isolated segment of the plasmodial strand, we constructed an electromagnetic tensiometer with a sensitivity of $0.1 \mathrm{mg}(6,7,8)$. As shown in Fig. 1, a plasmodial strand segment was set vertically on the tensiometer with one terminal connected to the glass

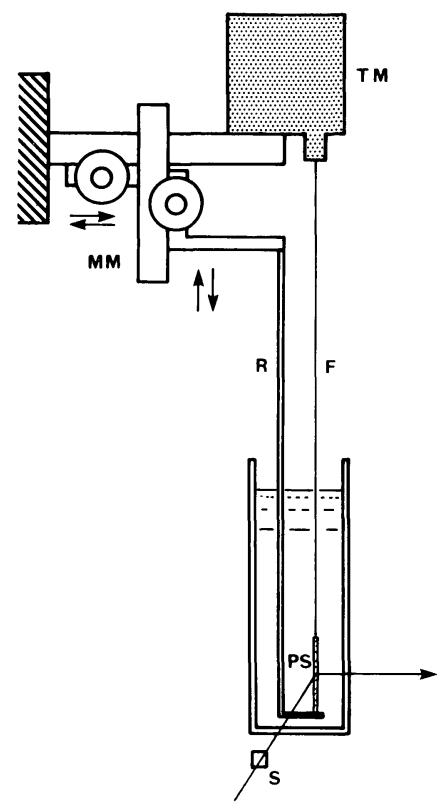

Fig. 1. Setup for simultaneous measurement of isometric tension production and cytoplasmic pH of a plasmodial strand segment. PS: plasmodial strand loaded with fluorescein. TM: tensiometer. MM: micromanipulator. S: slit. R: glass rod. F: glass fiber. 
fiber hanging from the tensiometer and the other terminal connected with the glass rod affixed to the micromanipulator. Cyanoacrylate, a surgical adhesive agent ("Aron Alpha," Toa Gosei Kagaku Co. Ltd., Tokyo) was used to connect the strand ends to the glass fiber and rod.

Fluorescence measurements of the plasmodial strand were conducted by submerging the strand in $3 \mathrm{mM}$ Mes buffer ( $\mathrm{pH}$ 6.0) in a standard cuvette. A dual beam difference spectrofluorometer (Model RFO 520 Shimazu, Kyoto) was used for the experiments. Excitation beams irradiated the middle region of the strand between the upper and lower terminals after passing through a slit of $2 \mathrm{~mm}$ (vertical) $\times 4 \mathrm{~mm}$ (horizontal). All the experiments were done at room temperature $\left(20-22^{\circ} \mathrm{C}\right)$.

\section{RESULTS}

$p H$ dependence of fluorescein spectra. At $\mathrm{pH} 6$ or higher the excitation spectra of the fluorescein solutions (Em $560 \mathrm{~nm}$ ) were dominated by a large peak at an excitation wavelength of about $490 \mathrm{~nm}$, as shown in Fig. 2. At lower $\mathrm{pH}$, this peak became smaller than another peak at around $470 \mathrm{~nm}$. A pH-insensitive excitation wavelength was found at $448 \mathrm{~nm}$ for the range of $\mathrm{pH} 6-8$. Thus, as shown in Fig. 3, the $\mathrm{pH}$ in this range could be sensitively measured by the ratio of fluorescence intensities excited at $490 \mathrm{~nm}$ and $448 \mathrm{~nm}$, which will be referred to as the $490 / 448$ (Em 560) ratio. This ratio is independent of the concentration of the fluorescent dye and the volume of the solution $(4,11)$. Hence the $\mathrm{pH}$ can be estimated from this ratio without knowing the fluorescein concentration.

Fluorescein-loaded plasmodium. The plasmodium loaded with fluorescein exhibited no apparent abnormalities in morphology or behavior except that it appeared greenish. When it was left on the agar surface, it moved about for as long as one week and eventually developed fruit bodies. Segments of a fluorescein-loaded plasmodial strand generated the normal rhythmical tensile force under isometric conditions. The amount of fluorescein trapped within the plasmodium was less than $0.2 \mathrm{mM}$,

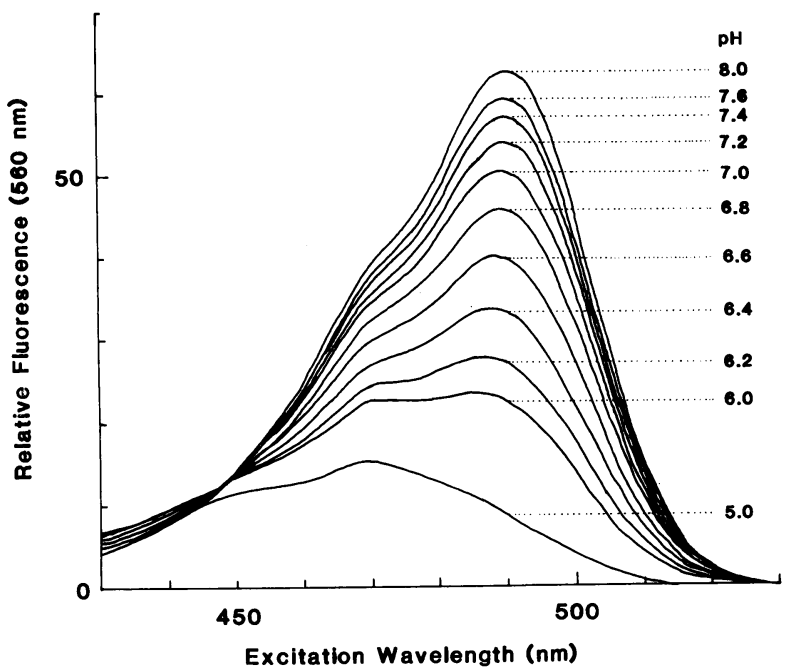

Fig. 2. Fluorescence excitation spectra of fluorescein solution at various pHs monitored at the emission peak of $560 \mathrm{~nm}$. 


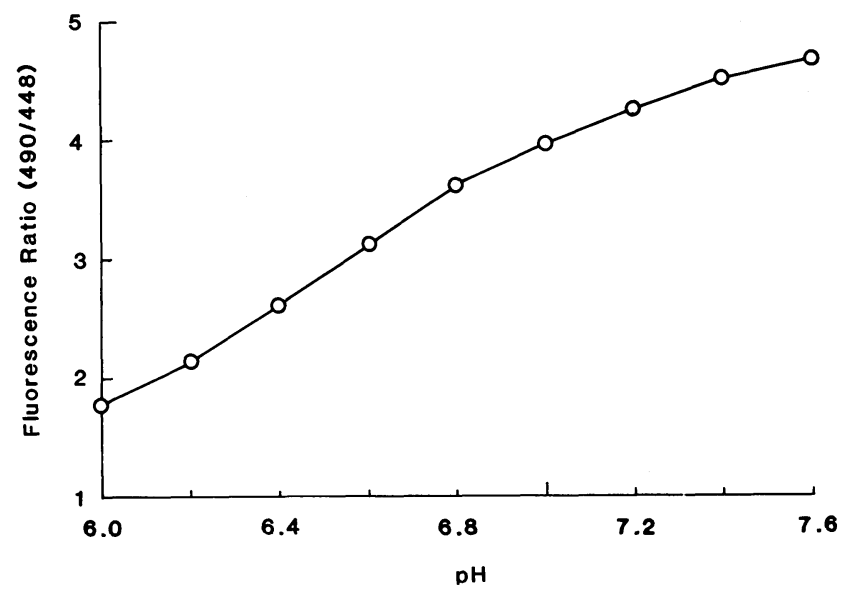

Fig. 3. Relationship between fluorescence ratio $(490 / 448, \mathrm{Em} 560 \mathrm{~nm})$ and $\mathrm{pH}$ of the fluorescein solution.

as determined from the supernatant after the centrifugation $(100,000 \mathrm{~g}$ for $1 \mathrm{~h})$ of homogenized plasmodia in $0.1 \mathrm{M}$ phosphate buffer solution.

Spectral characteristics of fluorescein in the plasmodium. The excitation spectrum of the plasmodial strand in which fluorescein was trapped had a large peak at about $493 \mathrm{~nm}$ (a in Fig. 4), which meant that the peak had shifted from that of the fluorescein solution toward the red region. Our experimental system did not allow us to obtain spectra from the same plasmodial strand before and after fluorescein loading, so we determined the average spectrum of the native plasmodial strands statistically ( $b$ in Fig. 4). As the curve had a sufficiently constant profile from one sample to another $(530 / 493 / 448=1.00 /(0.668 \pm 0.042) /(0.497 \pm 0.086)$ for 57 specimens $)$, we adopted it as the profile of the control before the fluorescein loading. The spectrum of the native plasmodial pigments had no distinct peak at $493 \mathrm{~nm}$. Since the fluorescence of the fluorescein solution reached zero as the excitation wavelength was increased to

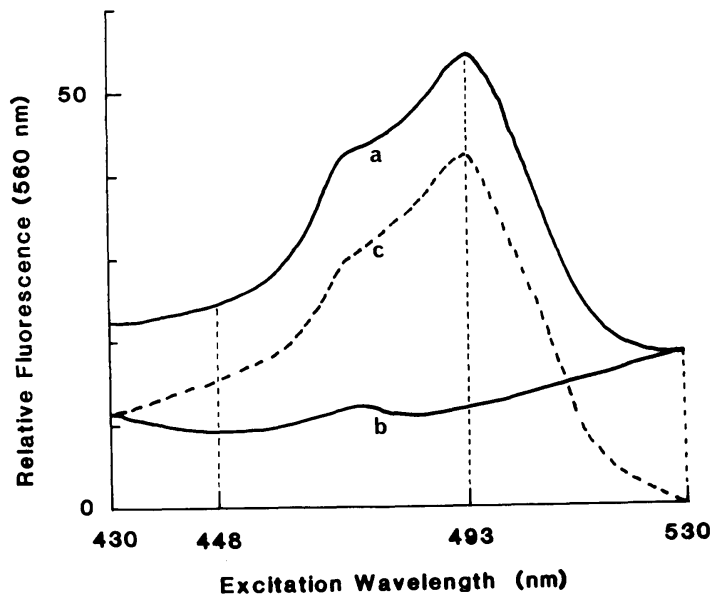

Fig. 4. Fluorescence excitation spectra of the plasmodial strand. a: strand loaded with fluorescein diacetate. $b$ : natural, unstained strand (control). $c$ : difference spectrum between $a$ and $b$. 


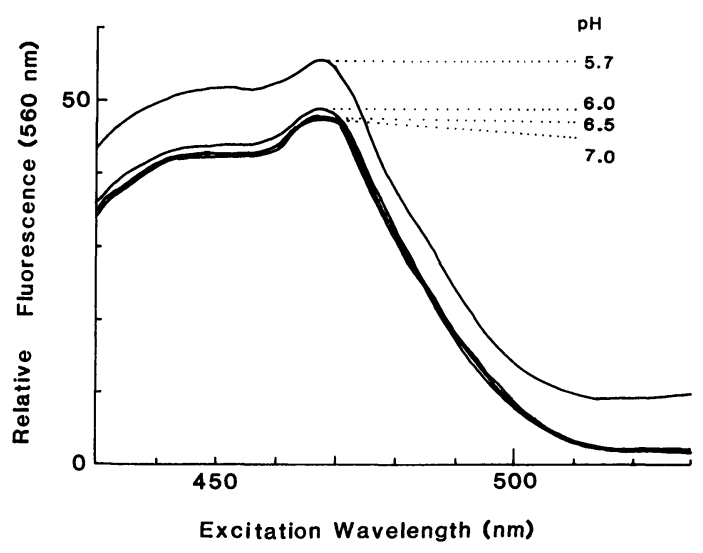

Fig. 5. Fluorescence excitation spectra of the native fluorescent pigments in the supernatant of the plasmodium homogenized in $0.1 \mathrm{M}$ phosphate buffer $(\mathrm{pH} \mathrm{6.8)}$ of 10 times the volume and centrifuged subsequently at $100,000 \mathrm{~g}$ for $1 \mathrm{~h}$. pH change was controlled at 7.0, 6.5, 6.0 and 5.7.

$530 \mathrm{~nm}$ (cf. Fig. 2), the fluorescence (a) excited by $530 \mathrm{~nm}$ should be equal to the fluorescence of the native strand system. Thus, the level and magnitude of spectrum (b) were determined.

To estimate the intracellular $\mathrm{pH}$, we had to obtain the 493/448 (Em 560) ratio from the spectrum of the fluorescein trapped within the plasmodial strand; the spectrum of the intact, unstained strand (b, in Fig. 4) was subtracted from the total spectrum (a), and the difference spectrum (c) between (a) and (b) was then assumed to represent the spectrum of only the fluorescein dye trapped within the plasmodial strand. Native pigments in the supernatant after homogenization of the plasmodium in $0.1 \mathrm{M}$ phosphate buffer and centrifugation $(100,000 \mathrm{~g}$ for $1 \mathrm{~h})$ had no $\mathrm{pH}$ sensitivity in the range of excitation wavelengths studied (Fig. 5).

Cytoplasmic $\mathrm{pH}$ changes and force production. The fluorescein-loaded plasmodial strand was set on the tensiometer and held vertically in the standard fluorescence cuvette filled with a weakly buffered solution ( $\mathrm{pH} \mathrm{6.0).} \mathrm{As} \mathrm{mentioned} \mathrm{before,} \mathrm{a} \mathrm{small}$ area in the middle part of the strand was irradiated with an excitation beam, and then changes in tension output and fluorescence intensities were recorded continuously and simultaneously with a pen chart recorder. However, since the thickness of the strand during the contraction and relaxation cycle was not exactly constant even under isometric conditions, the extent of the changes in fluorescence intensity which were due to the $\mathrm{pH}$ changes per se had to be determined.

To detect the intracellular $\mathrm{pH}$ changes independently of the changes in strand thickness, the fluorescences due to excitation at $493 \mathrm{~nm}$ and $448 \mathrm{~nm}$ were measured alternately at about $10 \mathrm{sec}$ intervals. Cyclic changes in 448-nm-excited fluorescence were $\mathrm{pH}$ independent and showed only mass changes. As shown in Fig. 6, the 448nm-excited fluorescence (B) was low, and the magnitude of its rhythmic fluctuation was small. To eliminate the effect of mass changes, the 448-nm-excited fluorescence was subtracted from the 493-nm-excited fluorescence. This normalized the average intensity level of the fluorescence excited at $448 \mathrm{~nm}$ (B) to that of the fluorescence excited at $493 \mathrm{~nm}$ (A), with the cyclic fluctuations amplified by the same factor. If the intracellular $\mathrm{pH}$ did not change in association with force production, the two 


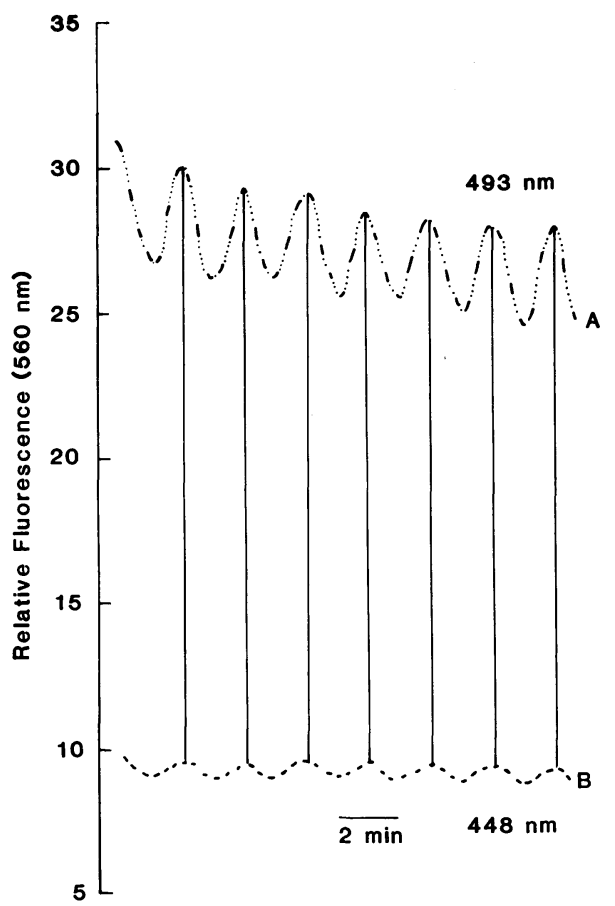

Fig. 6. Alternate recordings of cyclic changes in fluorescence excited at 493 (A) $\mathrm{nm}$ and 448 (B) $\mathrm{nm}$ with a period of $10 \mathrm{sec}$.

undulating curves of fluorescence excited at 448 and $493 \mathrm{~nm}$ should have overlapped with each other and there would be no difference in amplitude between the two waves. However, if the intracellular $\mathrm{pH}$ did change in association with force production, there would be a difference in the wave amplitude between the two curves.

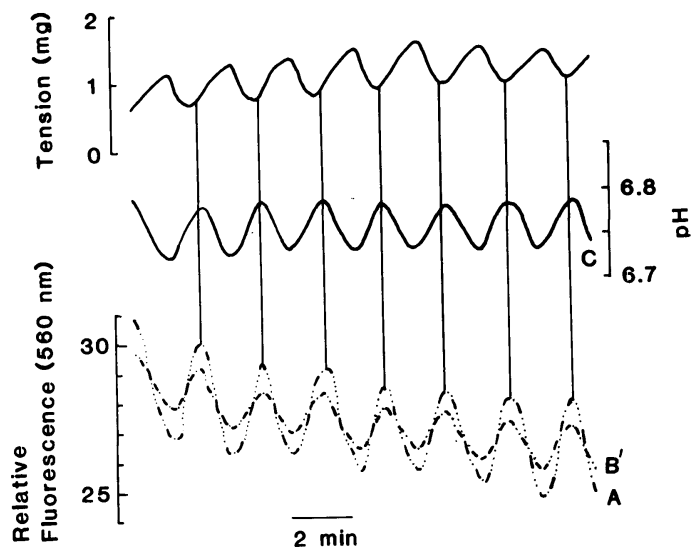

Fig. 7. Oscillations in cytoplasmic $\mathrm{pH}$ and tension development in a plasmodial strand. A: fluorescence change excited at $493 \mathrm{~nm}$. B': fluorescence change excited at $448 \mathrm{~nm}$ and normalized to the level of A. C: difference in fluorescence between A and B'. This can be converted into changes in $\mathrm{pH}$ (cf. Figs. 3, 4, and 6). 
In Fig. 7, B' represents the 448-nm-excited fluorescence (B in Fig. 6) normalized to the same level as A with proportional amplification of the fluctuations. Curve $\mathrm{C}$ shows the difference between A and B'. The temporal changes in the 493/448 (Em 560) ratio of the intracellular fluorescein could be followed from curve $C$. The cytoplasmic $\mathrm{pH}$ could be calibrated by adopting the 490/448 (Em 560) ratio of fluorescein solution (Fig. 3) for the intracellular fluorescein (fluorescence ratio: 493/448). The $\Delta \mathrm{pH}$ was calibrated likewise. By this procedure the fluctuation of the cytoplasmic $\mathrm{pH}$ was found to be in the range of 6.7-6.8 (Fig. 7). Since the 490/448 (Em 560) ratio is almost a linear function of $\mathrm{pH}$ in the range of $\mathrm{pH} \mathrm{6.0-7.0,} \mathrm{the} \mathrm{wave} \mathrm{form} \mathrm{representing}$ intracellular $\mathrm{pH}$ fluctuations was practically the same as that of the difference spectrum $\mathrm{C}$. The intracellular $\mathrm{pH}$ averaged $6.6 \pm 0.5$ for the 34 specimens.

To sum up, the $\mathrm{pH}$ oscillations had the same period as that of the tensile force oscillations, but the two were in antiphase to each other; i.e., the phases of maximal tension corresponded to those of the cytoplasmic-pH minima and the phases of minimal tension (relaxation phase) to those of cytoplasmic-pH maxima. The $\mathrm{pH}$ oscillation amplitude was $0.13 \pm 0.06 \mathrm{pH}$ unit (for 34 specimens).

\section{DISCUSSION}

An attempt was made to find a possible correlation between $\mathrm{pH}$ changes and the tensile force production in a plasmodial strand segment. To measure cytoplasmic $\mathrm{pH}$, we adopted a fluorometric method using fluorescein diacetate as a $\mathrm{pH}$ indicator. It was difficult to apply conventional $\mathrm{pH}$ microelectrode to Physarum plasmodium because the electrode tip was quickly resealed after insertion into the cytoplasm.

There is a long-term variation of intracellular $\mathrm{pH}$ that corresponds to the period of the cell cycle, with a low point at mid-interphase and a peak at mitosis $(3,9)$. The intracellular fluctuation over the cell cycle was reported to be between 6.0 and 6.6 (3), or between 7.0 and 7.5 (9). Our experiments, in which plasmodial strands at random stages of the cell cycle were used, showed that the average intracellular $\mathrm{pH}$ was $6.6( \pm 0.5)$.

It was previously reported that the concentrations of ATP and $\mathrm{Ca}^{2+}$ change cyclically with the same period as cyclic tension development $(16,17)$. However, we ascertained that fluorescence intensity of fluorescein was insensitive to $\mathrm{Ca}^{2+}(\mathrm{pCa} 6-8)$ and ATP $(0-1 \mathrm{mM})$ (data not shown). Thus intracellular fluorescein in the plasmodium should reflect the real $\mathrm{pH}$ change occurring in the cytoplasm or in both cytoplasm and mitochondria $(13,14)$.

There is evidence to show that increase in $\mathrm{pH}$ induces polymerization of actin. For instance, cortices isolated from the unfertilized sea urchin egg at $\mathrm{pH}$ 6.5-6.7 do not contain filamentous actin, while those isolated at $\mathrm{pH} 7.3-7.5$ develop a large number of microvilli which contain bundles of actin filaments (1). The profilactin in the sperm acrosomal vacuole of a starfish and other animals is transformed into filaments at $\mathrm{pH} 7.8-8.2$. However, at $\mathrm{pH}$ 6.0-6.5 there is no polymerization of the actin (15). Thus the intracellular $\mathrm{pH}$ may also control the polymerization-depolymerization of actin in the plasmodium during the contraction-relaxation cycle. These observations appear to be inconsistent with ours, but it should be noted that cyclic tension development of the plasmodial strand segment is coupled with cyclic changes in the aggregation pattern of F-actin and not with G-F transformation of actin. According to Nagai et al. (10), the assembly of the microfilaments and their 
higher order organization are characteristic of the phase of the contraction-relaxation cycle; hence they must also be correlated with cytoplasmic pH. Our data and observations made by Nagai et al. indicate that cytoplasmic $\mathrm{pH}$ decreases in the contracting phase when microfilaments form compact straight bundles, and it increases in the relaxing phase when these bundles are loosened and each filament composing them becomes flexible.

Although the causal relationship between the cytoplasmic $\mathrm{pH}$ and the microfilament morphology still remains to be dealt with, it may be said on the basis of our present observations and those made by Nagai et al. (10) that the lower pH should favor the assembly of microfilaments in straight compact bundles and the higher $\mathrm{pH}$ the loosening of their bundle structure.

\section{REFERENCES}

1. BegG, D.A. and L.I. ReBhun. pH regulates the polymerization of actin in the sea urchin egg cortex. J. Cell Biol. 83, 241-248, 1979

2. Camp, W.G. A method of cultivating myxomycete plasmodia. Bull. Torrey Bot. Club. 63, 205210,1936

3. Gerson, D.F. and A.C. Burton. The relation of cycling of intracellular pH to mitosis in the acellular slime mould Physarum polycephalum. J. Cell Physiol. 91, 297-304, 1977

4. HeIPLE, J.M. and D.L. TAYLOR. Intracellular pH in single motile cells. J. Cell. Biol. 86, 885-890, 1980

5. Hirose, T., T. Ueda and Y. Kobatake. Changes in intracellular pH accompanying chemoreception in the plasmodia of Physarum polycephalum. J. Gen. Microbiol. 128, 2647-2651, 1982.

6. KamiYa, N. Contractile properties of the plasmodial strand. Proc. Japan Acad. 46, 1026-1031, 1970

7. KamiYa, N. and Y. Yoshimoto. Dynamic characteristics of the cytoplasm. A study on the plasmodial strand of a myxomycete. in Aspects of Cellular and Molecular Physiology, ed. Hamaguchi, K. Univ. Tokyo Press, Tokyo, pp. 167-189, 1972

8. Kamiya, N., R.D. Allen and R. Zeh. Contractile properties of the slime mold strand. Acta Protozool. 11, 113-124, 1972

9. Morisawa, M. and R.A. Steinhardt. Changes in intracellular pH of Physarum plasmodium during the cell cycle and in response to starvation. Exp. Cell Res. 140, 341-351, 1982

10. Nagai, R., Y. Yoshimoto and N. KamiYa. Cyclic production of tension force in the plasmodial strand of Physarum polycephalum and its relation to microfilament morphology. J. Cell Sci. 33, 205-225, 1978

11. Ohкuma, S. and B. Poole. Fluorescence probe measurement of the intralysosomal $\mathrm{pH}$ in living cells and the perturbation of $\mathrm{pH}$ by various agents. Proc. Natl. Acad. Sci. USA. 75, 3327-3331, 1978

12. Seifriz, W. and M. Zetzmann. A slime mould pigment as indicator of acidity. Protoplasma 23, 175-180, 1935

13. ThOmas, J.A., P.C. KolbeK and T.A. LANGwORTHY. Spectrophotometric determination of cytoplasmic and mitochondrial $\mathrm{pH}$ transitions using trapped $\mathrm{pH}$ indicators. in Intracellular pH: Its Measurement, Regulation, and Utilization in Cellular Functions. ed. Nuccitelli, R. and D.W. Deamer, Alan R. Liss. Inc. New York, pp. 105-123, 1981

14. Thomas, J.A., R.N. BuchsBaum, A. ZimNiaK and E. RaCKer. Intracellular pH measurements in Ehrlich ascites tumor cells utilizing spectroscopic probes generated in situ. Biochem. 18, 2210-2218, 1979

15. Tilney, L.G., D.P. Kiehart, C. Sardet and M. Tilney. Polymerization of actin. IV, Role of $\mathrm{Ca}^{++}$and $\mathrm{H}^{+}$in the assembly of actin and in membrane fusion in the acrosomal reaction of echinoderm sperm. J. Cell Biol. 77, 536-550, 1978 
16. Yoshimoto, Y., F. Matsumura and N. KamiYa. Simultaneous oscillations of $\mathrm{Ca}^{2+}$ efflux and tension generation in the permealized plasmodial strand of Physarum. Cell Motility, 1, 433443, 1981

17. Yoshimoto, Y., T. Sakai and N. KamiYa. ATP oscillation in Physarum plasmodium. Protoplasma, 109, 159-168, 1981

(Received for publication, March 26, 1985) 